\title{
Loss of vision before ophthalmic referral in blind and partially sighted diabetics in Bristol
}

Bristol Eye Hospital,

Bristol

J B Clark

R H B Grey

University of Bristol Department of Medicine, Bristol Royal Infirmary

K K T Lim

Department of Medicine, Frenchay Hospital,

Bristol

C J Burns-Cox

Correspondence to:

R H B Grey, Lower Maudlin

Street, Bristol BS1 2LX.

Accepted for publication

1 July 1994

\author{
J B Clark, R H B Grey, K K T Lim, C J Burns-Cox
}

\begin{abstract}
Data from all patients registered blind from diabetic retinopathy in Avon during a 16 month period were analysed with regard to management before hospital referral. The main findings were: $50 \%$ of the patients had no screening for retinopathy and were known to be diabetic; $25 \%$ were regularly screened for retinopathy (three quarters by local opticians); $22 \%$ were newly diagnosed as diabetic at the time of hospital referral. The degree of visual loss at the time of first hospital attendance was found to be marked (average 4.4 Snellen lines of acuity) but was not significantly different for different sources of referral. Only one eye of one patient had normal acuity at first attendance and $88 \%$ had lost two or more lines; $72 \%$ of registrations were a result of diabetic maculopathy. Delay from waiting for hospital appointments did not contribute significantly to the outcome in the group of patients studied. (Brf Ophthalmol 1994; 78: 741-744)
\end{abstract}

The benefits of laser photocoagulation in the treatment of diabetic maculopathy and proliferative diabetic retinopathy are well established. ${ }^{1-11}$ It is also widely accepted that with respect to diabetic maculopathy the maximum benefit of treatment is obtained if treatment is begun before any loss of vision has occurred..$^{12} 13$ The identification of diabetics at risk of losing vision is, therefore, of crucial importance if the population of diabetics is to benefit maximally from current treatment practices. Probably as a result of both the increasing incidence of diabetes and greater longevity of the population the prevalence of registerable blindness is not decreasing. ${ }^{14}$

We wished to study those diabetics who were ultimately registered blind to see whether screening had taken place and also to investigate which factors may have contributed to loss of vision that is, (1) whether there was late referral to an ophthalmologist, (2) if delay had occurred in implementing hospital treatment, or (3) whether visual loss progressed in spite of prompt treatment.

\section{Methods}

We reviewed all the BD8 registrations from July 1990 to October 1991 for the hospitals serviced by the consultant staff attached to the Bristol Eye Hospital. We examined the medical records of these patients to find those who had been registered blind or partially sighted because of diabetes (visual acuity of $6 / 36$ or worse). We reviewed the original referral letters, including the optometrists' report if this was available. The general practitioner records were also examined for details of diabetes, information about the referral and eye care. The date of referral, the reason and, if possible, the vision at the time of referral, were all noted. We then recorded the date of first visit to an ophthalmologist, the diagnosis and vision at this visit, and compared these with the referral details, with particular respect to the accuracy of referral, the delay from referral to first visit, and the level of visual loss at the first visit. All referral letters were reviewed by one doctor (JBC) and assessed as to whether the information provided suggested an urgent, soon, or routine appointment should have been given.

Where information from general practitioners' notes was unclear about eye care, the patients were either written to or telephoned, to see if they had had their eyes examined before their referral to an ophthalmologist. Specifically they were asked if they regularly - that is, yearly, 'had a bright light shone in to check the back of the eyes'.

Levels of vision were recorded in standard Snellen format. For the purposes of statistical calculation the Snellen acuities were converted to $\log$ MAR format ${ }^{15}$ and allowances made for intereye correlation. ${ }^{16}$ Comparisons of acuities of patients were made between the various sources of referral - that is, general practitioners, optometrists via general practitioners, hospital diabetic physicians, or tertiary referrals from other ophthalmologists. Similar calculations were also performed comparing previously screened with unscreened patients. For comparisons between the two groups $t$ tests were used, and for comparisons between three groups analysis of variance was used.

\section{Results}

There were $572 \mathrm{BD} 8$ registrations. We were able to review the records of 471 of these patients. The main reasons for being unable to review patients' records was that they were unavailable or were kept at other hospitals. There were 48 diabetics, of whom 32 were registered principally because of diabetic retinopathy; in the remaining 16,13 were registered for age-related macular degeneration; one was from macular hole; one from myopic degeneration; and one from cataract. The ages of the patients ranged from 21 to 90 years, with a mean of $68 \cdot 7$ (Fig 1). Two patients had typical juvenile onset diabetes, one had insulin dependent diabetes occurring at 40 years of age, while the rest had maturity onset diabetes. Of the latter group, four patients were on insulin. There was a preponderance of females with only 11 of the 32 being male. The average duration of known diabetes was 6.8 years with a range from newly diagnosed to 28 years. Seven $(22 \%)$ of the patients were newly diagnosed at the time of 


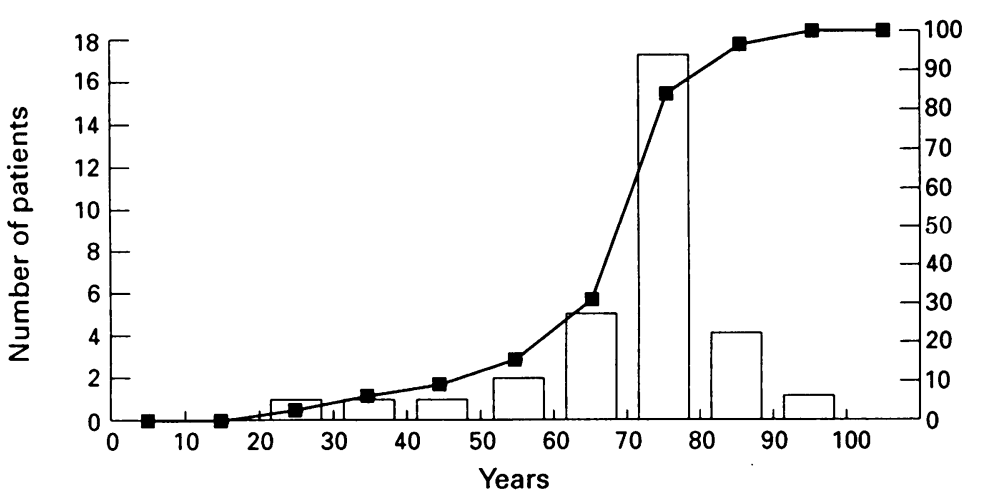

Figure 1 Age distribution at hospital attendance. $\square=$ Cumulative percentage of patients. referral, six by general practitioners and one when seeing the ophthalmologist. The reasons for poor visual acuity in all eyes are given in Table 1.

Of the 32 patients, eight (25\%) had regular annual eye checks before referral to an ophthalmologist (six were checked by optometrists and two by a physician). Sixteen (50\%) known diabetics had no regular eye checks, and in the remaining patients, seven (22\%) were not known to be diabetic and in one it was not clear whether or not screening took place.

The average wait for an appointment to see an

Table 1 Reasons for visual loss

\begin{tabular}{lr}
\hline Reason for BD8 listing & $\begin{array}{l}\text { Number of } \\
\text { eyes }(\%)\end{array}$ \\
\hline Maculopathy & $46(71 \cdot 8)$ \\
Traction detachment & $7(10 \cdot 9)$ \\
Rubeotic glaucoma & $2(3 \cdot 1)$ \\
Refused surgery & $4(6 \cdot 3)$ \\
Expulsive haemorrhage & $1(1 \cdot 6)$ \\
Amblyopia & $1(1 \cdot 6)$ \\
Unexplained & $2(3 \cdot 1)$ \\
Awaiting surgery & $1(1 \cdot 6)$ \\
Total & $64(100)$ \\
\hline
\end{tabular}

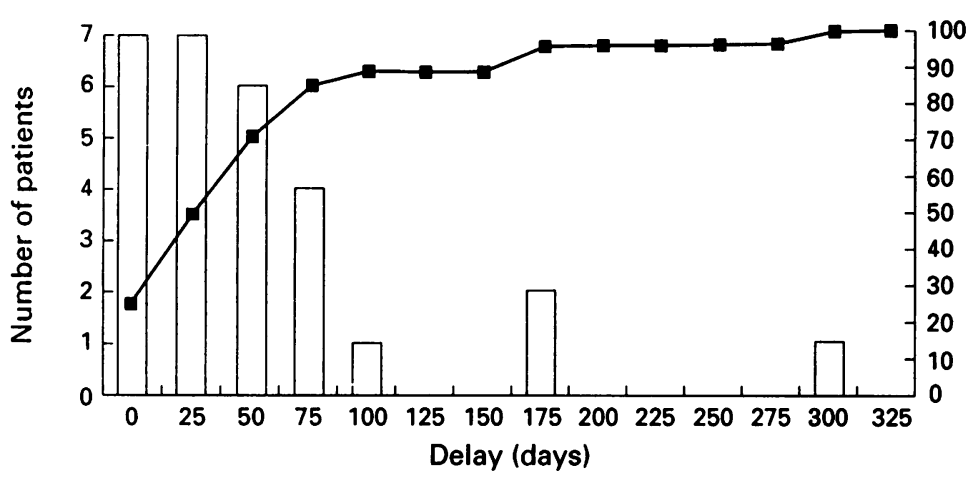

Figure 2 Delay from general practitioner referral to first hospital visit (days).

$\mathbf{q}=$ Cumulative percentage of patients.

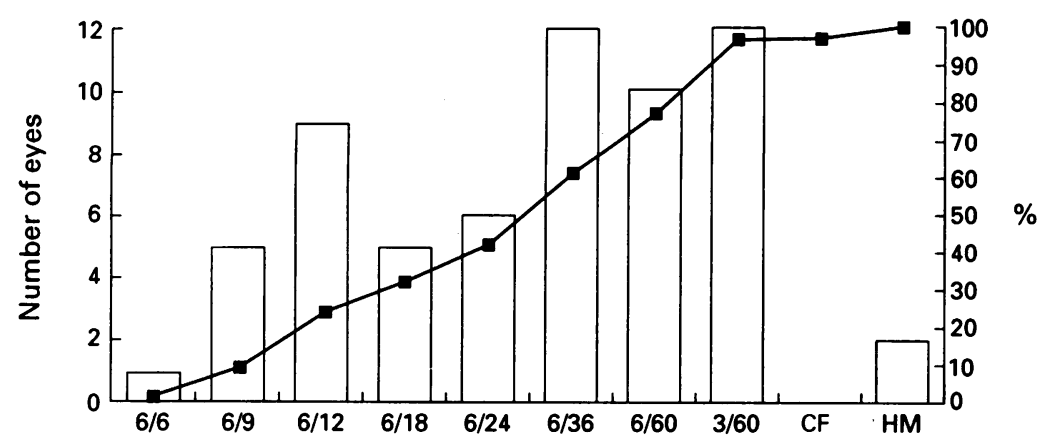

Figure 3 Visual acuity at first hospital visit (eyes). $C F=$ counting fingers; $H M=$ hand movements; $\mathbf{D}=$ cumulative percentage of eyes. ophthalmologist was 64.5 days from the time of the referral letter. Figure 2 shows that over $70 \%$ of patients were seen within 75 days of referral. Of the patients who waited over 100 days two were inaccurate referrals (true diagnoses were maculopathy) and two were accurate referrals that should have been seen sooner (both had maculopathy). The patient who waited over 300 days had an inaccurate referral diagnosis of cataracts.

Of the total number of 64 eyes, $37(58 \%)$ were referred with an accurate diagnosis, 23 (36\%) were inaccurate, and in four $(6 \%)$ the referral details were not available. In 14 of the 23 inaccurate referrals there had been failure to recognise either sight reducing maculopathy or retinopathy at all. There was no difference between the inaccurate and accurate referrals with respect to loss of visual acuity at first visit, with both groups having lost 4.4 lines of vision.

The average loss of visual acuity in the group as a whole at their first visit to an ophthalmologist was 4.4 lines in each eye. Figure 3 shows the distribution of visual acuities for the 64 eyes at first hospital presentation. The average visual acuity in the group having regular eye checks was $6 / 30$ and the average acuity in the group not having regular eye checks was $6 / 39$. This is not a statistically significant difference $(p=0.395)$. Only one eye had normal visual acuity at the time of first examination in hospital. Five eyes $(8 \%)$ were $6 / 9$, nine eyes $(14 \%)$ were $6 / 12$, but the remaining $49(77 \%)$ were $6 / 18$ or worse.

The referral letters that were deemed by one of us (JBC) to suggest an urgent problem had to wait, on average, 32 days to see an ophthalmologist. There were 11 patients in this group, two of whom had to wait more than 90 days. It is not possible to tell whether this delay was responsible for any loss of vision as the visual acuity at the time of referral was not recorded. There were 15 patients whose letters suggested a semi-urgent problem and these waited, on average, 55 days for an appointment. For the routine referrals the average wait was 160 days.

Those considered to be urgent or semi-urgent referrals had a visual acuity average of $6 / 30$ compared with an average $6 / 34$ for the nonurgent group ( $p=0.708$ : not significant). The additional average waiting time of 3 to 4 months for the 'routine' cases did not seem to have an adverse effect on presenting visual acuity.

The visual acuities of patients from different referral sources were compared and are given in Table 2. The visual acuities for patients referred by general practitioners alone had significantly worse vision than those whose referrals were initiated by optometrists or hospital physicians $(p=0.0004)$. Three cases were tertiary referrals for management of advanced diabetic eye disease. All three patients had severe visual loss

Table 2 Average visual acuities by source of referral

\begin{tabular}{lll}
\hline & $\begin{array}{l}\text { Snellen } \\
\text { acuity }\end{array}$ & Log MAR \\
\hline Optician & $6 / 20$ & 0.5141 \\
Hospital physician & $6 / 23$ & 0.5807 \\
General practitioner & $6 / 51$ & $0.9317 \star \star$ \\
\hline
\end{tabular}

${ }^{\star \star} \mathrm{p}=0 \cdot 0004$. Three tertiary referrals excluded. 
at the time of referral (acuities $3 / 60,3 / 60 ; 6 / 36$, $6 / 60 ; H M, H M)$.

\section{Discussion}

Care must be exercised when drawing conclusions from analysis of blind registration forms. Not all eligible diabetics will be registered and because registration requires bilateral visual loss, ocular morbidity from retinopathy could be markedly underrated. However, several points were raised by this study with regard to management of diabetic retinopathy and screening.

Eighty per cent of patients were examined by an ophthalmologist within 2 months of referral to hospital, and over $90 \%$ by 3 months. Therefore, blindness in this population did not seem to be a result of delayed hospital attention in the majority of cases. The finding of a considerable loss of acuity (average 4.4 lines) before the time of hospital attendance suggests that those who ultimately finish up with severe visual loss in both eyes have well advanced retinopathy before hospital referral. The average acuities of the urgent and semi-urgent cases were not significantly different from the routine cases even though the latter waited an additional 3 months before being seen in hospital. Seventy two percent of patients had maculopathy which is recognised as only slowly progressive, so earlier referral should have been possible. With the knowledge that over $80 \%$ of cases of proliferative retinopathy and maculopathy are reversible by photocoagulation, particularly when identified early, it was disappointing to see how many individuals had marked visual loss before being seen.

It is sometimes assumed that retinopathy leading to blindness is of a more relentless nature and this study lends some support to this contention. Fifteen eyes had $6 / 12$ or better at hospital presentation but were later registerable. Within this group there were three eyes which had an acuity recorded at $6 / 9$ or better at the time of referral and had marked further deterioration in the relatively short waiting time before being seen in hospital. Four patients had bilaterally good vision (6/12 or better) when first seen but later went blind in spite of treatment. These cases suggest the retinopathy was progressive in spite of prompt attention. For the remaining patients, it is not possible to tell from this study whether earlier treatment would have saved blindness but it is likely that many of the early maculopathies would have been reversible. Late referral to an ophthalmologist may well have been a significant cause of poor outcome but this is not proved for all these cases in this study. The average acuity for the patients who waited for a routine appointment was not worse than those seen more urgently, which might suggest that deterioration was slow and could have been reversed earlier.

Evaluation of the efficacy of screening of retinopathy in the diabetic population cannot be deduced from this study. The large loss of acuity at the time of referral may reflect ineffective screening with late referral to an ophthalmologist, or may simply reflect retinopathy leading to blindness to be of a more aggressive nature in this group. All current methods of screening have a high rate of underestimating serious retinopathy - that is, poor sensitivity, but are good at identifying the presence or absence of any retinopathy - that is, good specificity. ${ }^{17}$ Difficulties arise in differentiating innocent background from serious retinopathy, particularly maculopathy which can be present for some time before affecting Snellen acuity. It was of interest to note that the number of optician referrals with visual loss was four times higher than for hospital physicians, whereas one might expect the latter to be monitoring the more uncontrolled or severe diabetics. There is no doubt that skilled physicians examining patients with dilated pupils provide an excellent screening mechanism for retinopathy. If opticians are used for screening, close supervision of acuity should be encouraged, probably at 6 monthly intervals, if any retinopathy at all has been observed. Referral should take place once any reduction of acuity is observed in the presence of retinopathy. Ideally patients with maculopathy should be referred before acuity has been affected. Parafoveal exudates can be identified easily by trained observers but macular oedema is much more difficult to see. Referral to an ophthalmologist should, therefore, follow if screeners observe any vascular changes within a disc diameter of the fovea, whether microaneurysms, haemorrhages, or exudates.

It has been recognised previously that maculopathy is nearly four times more common than proliferative retinopathy and in this study $72 \%$ of registrations were a result of maculopathy. These findings suggest that there is a proportion of diabetic patients who are suffering considerable visual loss and who could have been identified earlier in the course of their disease by simple visual acuity measurements.

The benefit of screening for retinopathy is well established $^{17-22}$ although debate continues with regard to the best method, whether by skilled observer or photography with acuity recordings. This study showed that $50 \%$ of those being registered had no regular screening of their retinas. However, there were nearly half as many blind registrations following regular screening, which suggests that serious retinopathy was not picked up quickly enough. Because the large preponderance of registrations $(72 \%)$ are from maculopathy any loss of acuity observed by a screener should be referred. Although recognising that proliferative retinopathy may not affect visual acuity initially, repeated acuity measurement is probably the single most significant step in identifying maculopathy and in trying to prevent blind registration. Whichever method of fundus examination is used for screening, it must be combined with acuity recording and particular attention to any retinopathy at all within a disc diameter of the fovea.

A further unexpected finding was that nearly a quarter of the study patients were not known to be diabetic before initiating their referral to hospital. Regular eye screening of this group, therefore, was not relevant, but perhaps these patients represent the best hope for reduction of future blindness from diabetic eye disease in accordance with the St Vincent's declaration. ${ }^{23}$ Early screening for diabetes would allow regular 
monitoring for the presence of retinopathy but this in itself poses major problems in health care delivery. Additional reductions in blind registration may also be achieved by improving standards of accuracy of referral to ophthalmologists (36\% inaccurate in this study) and by ensuring that delays in assessment in hospital do not occur once referral letters are received $(12 \%$ over 3 months in this study). Increasing the detail in referral letters, particularly with regard to acuity measurement, would also assist in categorising priority for the ophthalmologist.

The authors wish to thank Mrs L Clayton for typing the manuscript and to Mr John Sparrow for analysing the statistics.

1 Cheng $\mathrm{H}$. Response of proliferative diabetic retinopathy to xenon arc photocoagulation. Trans Ophthalmol Soc UK 1976; 96: 224-7.

2 Hercules BL, Gayed II, Lucas JB, Jeacock J. Peripheral retina ablation in the treatment of proliferative diabetic retinopathy: a three year intensive report of a randomised controlled study using argon laser. Br $\mathcal{F}$ Ophthalmol 1977; 61 $555-63$.

3 Diabetic Retinopathy Study Research Group. Photocoagulation treatment of proliferative diabetic retinopathy. Trans Am Acad Ophthalmol Otol 1978; 85: 82-106.

4 Diabetic Retinopathy Study Research Group. Photocoagulation treatment of proliferative diabetic retinopathy. Ophthal tion treatment of prolifera.
mology 1978; 85: 82-106.

5 Diabetic Retinopathy Study Research Group. Photocoagulation treatment of proliferative diabetic retinopathy. Ophthal mology 1981; 88: 583-600.

6 Townsend C, Bailey J, Kohner E. Xenon arc photocoagulation for the treatment of diabetic maculopathy. Brf Ophthalmo 1980; 64: 385-91.

7 Reeser F, Fleischman J, Williams GA, Goldman A. Efficacy of argon laser photocoagulation in the treatment of circinate argon laser photocoagulation in the treatment of circinatic
diabetic retinopathy. Am $\mathcal{F}$ Ophthalmol 1981; 92: 762-7.
8 British Multicentre Study Group. Photocoagulation for proliferative diabetic retinopathy: a randomised controlled clinica trial using the xenon arc. Diabetologia 1984; 26: 109-15.

9 Stenkula S. Photocoagulation in diabetic retinopathy: a multicentre study in Sweden. Acta Ophthalmol 1984; 162 (suppl): $1-100$

10 Early Treatment Diabetic Retinopathy Study Research Group. Photocoagulation for Diabetic Macular Oedema. Early Treatment Diabetic Retinopathy Study Report, num ber 1. Arch Ophthalmol 1985; 103: 1796-806.

11 Grey RHB. The treatment of diabetic maculopathy by argon laser photocoagulation. Trans Ophthalmol Soc UK 1986; 104 424-30.

12 British Multicentre Study Group. Photocoagulation for diabetic maculopathy: a randomised controlled clinical tria using the xenon arc. Diabetes 1983; 32: 1010-6.

13 Early Treatment of Diabetic Retinopathy Study. Report 9. Ophthalmology 1991; 98: 766-85.

14 Grey RHB, Burns-Cox CJ, Hughes A. Blind and partial sight registration in Avon. Br f Ophthalmol 1989; 73: 88-94.

registration in Avon. Br F Ophthalmol 1989; 73: 88-94.
15 Holladay JT, Prager' TC. Mean visual acuity. Am $\mathcal{F}$ Ophthalmol 1991; 111: 372-4.

16 Rosner B. Statistical methods in ophthalmology; an adjustment for the intraclass correlation between eyes. Biometrics 1982; 39: 105-14.

17 Buxton MJ, Sculpher MJ, Ferguson BA, Humphreys JE Altman JFB, Spiegelhalter DJ, et al. Screening for treatable diabetic retinopathy: a comparison of different methods. Diabetic Medicine 1991; 8: 371-7.

18 Grey RHB, Morris A. Ophthalmic survey of a diabetic clinic: II Requirements for treatment. Br $\mathcal{F}$ Ophthalmol 1986; 70: $804-7$.

19 Williams R, Nussey S, Humphry R, Thompson G. Assessment of non-mydriatic fundus photography in detection of diaof non-mydriatic fundus photography in de
betic retinopathy. $B M \mathcal{F} 1986 ; 293: 1140-2$.

20 Jones D, Dolben J, Owens DR, Vora JP, Young S, Creagh FM. Non-mydriatic Polaroid photography in screening for diabetic retinopathy: evaluation in a clinical setting. $B M \mathcal{J}$ 1988; 296: 1029-30.

21 Rohan TE, Frost CD, Wald NJ. Prevention of blindness by screening for diabetic retinopathy: a quantitative assessment. BMF 1989; 299: 1198-201

22 Taylor R, Lovelock L, Tunbridge WMG, George K, Albert MM, Brackenridge RG, et al. Comparison of non-mydriatic retinal photography with ophthalmoscopy in 2159 patients: mobile retinal camera study. BMF 1990; 301: 1243-7.

23 Workshop Report. Diabetes care and research in Europe: The St Vincent's Declaration. Diabetic Medicine 1990; 7: 360. 